\title{
IoT Based Smart Home Automation for Windows using CoAP
}

\author{
Pravin Sunil Khillare ${ }^{1}$, Prof. Dr. Mrs. Dipashree M. Bhalerao ${ }^{2}$ \\ Dept. of Electronics \& Telecommunication, Sinhgad College of Engineering(SCOE), Pune, India ${ }^{1,2}$
}

\begin{abstract}
Internet of things(IoT) is smart network technique where smart devices or smart things connected through internet through IP address. IoT is smart standardization technology that provides stacks for machine-tomachine(M2M) communication. In the smart home the IoT devices controls \& monitors the electronics \& mechanical systems used in smart home. Mobile application is used for the interfacing of home devices with the user. The IoT allows the mobile device to control \& operate the household devices remotely from anywhere around the world using internet. For the interconnection between the device \& user a Wi-Fi model, Zig-Bee or a Bluetooth is used.

The sole purpose of this project is to develop a system that will provide remote control of home appliances and also provide security against the mishaps when the home host is not present, to assist the handicapped/old aged people at house through the use of internet.
\end{abstract}

Keywords: Internet of Things(IoT), Wi-Fi, Raspberry Pi, CoAP, Home Automation.

\section{INTRODUCTION}

The home automation refers to domestic environment that improves the quality of the resident life by facilitating a flexible, comfortable \& secured environment. Internet based home automation systems become the most popular home automation system, where the remote controlling and monitoring of a house appliances using internet.

For the remote connection between the user and the household devices internet is used, where with the use of Raspberry Pi controller the household devices \& the user is interconnected with an android application [1].

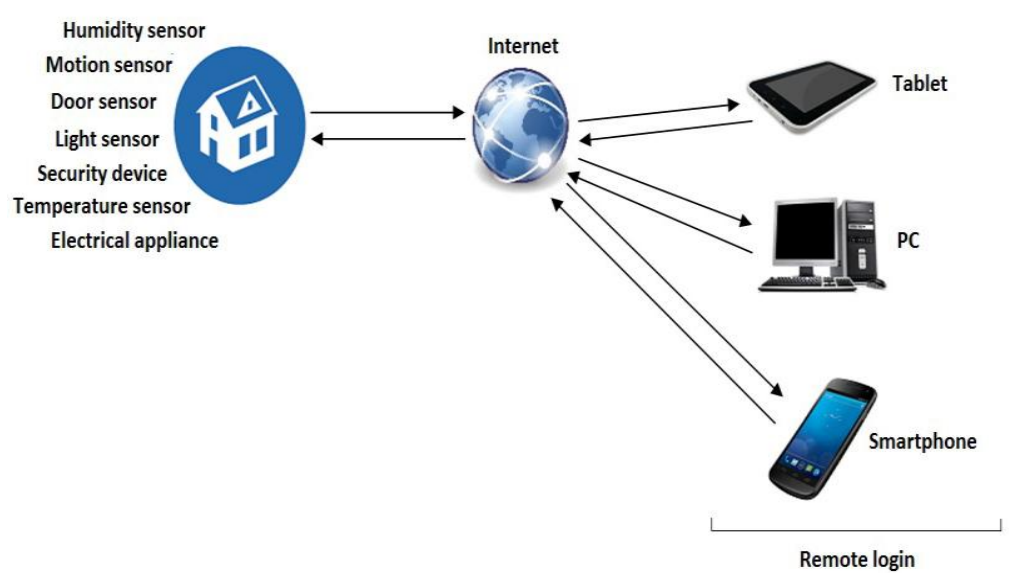

Fig 1.1 Proposed Home Automation System Over IoT

IoT is used for the establishment of virtual connection between

a hub \& a network controlling the electronic \& mechanical devices. The virtual connection is used to control, monitor $\&$ locate the status of the devices. IoT is the expansion of the internet services where the applications are operated under the use of internet with better convenient and higher precision values of their implementations. For the wireless communication, IoT provides device-to-device connections based on the Machine-to-Machine (M2M) protocol, providing fully duplex data transmission over the internet. For the home automation, we are using CoAP protocols of IoT which provides scalable, constrained applications. CoAP provides a lightweight fast asynchronous wireless data transmission architecture. Home automation over IoT using CoAP protocol is new terminology used in India, that reduces the bulkiness of the system, providing an easy system architecture with reduced instruction of set. CoAP is vastly usable because of its capability of reducing the complexity of system, providing better scalability \& susceptibility of system. For the wireless data transmission CoAP provides a reduced header, so the lightweight handshaking is done with reduced data instruction sets. 


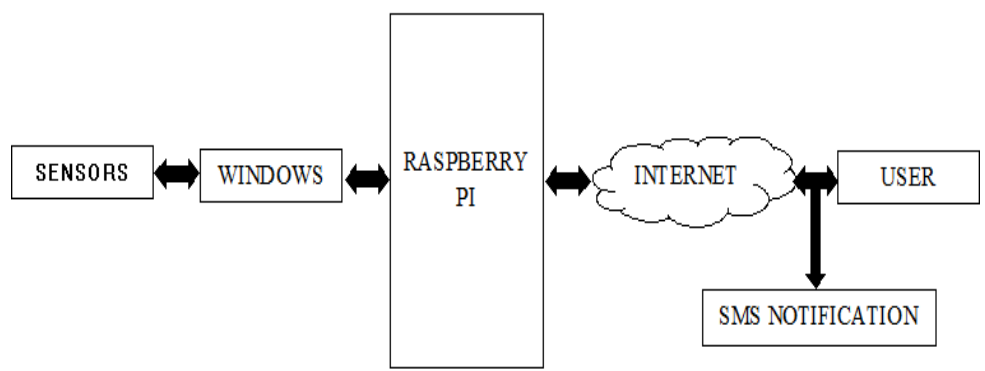

Fig. 1.2 Block Diag. of Home Automation over IoT

Presently Government of India (GoI) is developing 100 smart cities across the country which will create a huge demand for smart home automation in future. [2]

Smart home is the technology where the home appliances are monitored, controlled \& operated over internet. IoT is used for the interfacing of the home appliances \& the end user. This whole process of the monitoring \& controlling of household appliances over the internet using IoT is called as Smart Home in IoT environment or IoT based Smart Home. here windows are the integral home appliances that is to be operated \& monitored through internet. [3]

The main objective of this paper is to design \& implement a low cost, open source home automation system, which is capable of controlling \& automating windows of the smart home through the internet using smart devices. The main concern of this system is to assist the handicapped/old age people inside the home. [4]

\section{RELATED WORK}

In this paper we propose a smart home automation system that employs the integration of IoT, mobile device, cloud networking and wireless communication network to provide the end user with the remote control of windows within home.

For this system the end user doesn't have to worry about closing the windows or checking the burglar alarm, the user can check the status of household appliances from the database present online with the main server $\&$ change the status of the system accordingly.

This proposed system is used to support more secured, flexible, easily accessible \& more comfortable smart home system controlling \& monitoring windows using IoT over the internet. The implemented system is mainly built on the three components as: android device for end users, a Wi-Fi module, \& a Raspberry pi board. The Raspberry pi board is configured according to the service-oriented dynamic requirements of the smart home system. IoT is used for the wireless interference of the end user \& the Raspberry pi controller, through IP address. If there is presence of any intruder or breakage of glass or leakage of gas or change in intensity of light inside the house the system automatically alerts the user with a message to take any required action. This system helps operator in providing optimum security \& making the home smarter \& safer place to live in.

Home automation via IoT is preferred because of the low cost of android application, its immense popularity and ease of operation. The Raspberry pi board is also easily available at low cost and can be used for the controlling of multiple appliances inside the smart house with greater ease.

\section{Advantages of The Proposed System: -}

$>$ It provides better flexibility of operation \& long range operability of household appliances through the android application.

$>$ It has wide range of scalability.

$>$ It provides better security of home.

$>$ Increased energy efficiency.

$>$ Insight of home activities.

\section{III.IMPLEMENTATION DETAILS}

For the smart home automation system using IoT based CoAP protocol, a low cost Raspberry Pi microcontroller board alongside with an android application is used over the internet, for the controlling \& monitoring of windows. For the system Raspberry pi operate as intermediate, controlling both the software \& the hardware part of the system on board. As the communication in-between the end user application \& Raspberry pi is wireless, thus for the internetworking between them we have established a short range wireless network, to operate windows inside the smart home. For this 
home automation system as we are using IoT based CoAP protocol, the complexity of the set of operational instructions is reduced. CoAP provides easily integratable user datagram protocol (UDP) for the user application.

In this home automation system there is the use of various system devices are used, which are listed as,

\section{PIR Sensors}

It is connected with the windows from inside the home for the detection of motion, having $180^{\circ}$ angle of rotation with the range of operation up to 1-2 meter approximately. When any moving object comes within the range of PIR it detects the infrared radiation of the moving objects \& sends a burglar alarm to the end user.

\section{Motor Driver}

For the windows to shut down \& open after receiving response from end user a motor driver is used. It is connected with the hinges of the windows, allowing it to rotate in either direction i.e. clockwise \& anti-clockwise. Relay switches are used for changing the status of windows

3.Temperature sensors

Temperature sensors are used to check the real environmental conditions \& informs the user accordingly.

4. Gas Sensors

Burglar alarm with the gas sensors are used for the purpose of security inside the house, where there if any fire breakage or leaking of any toxic gas in the house, it sets the burglar alarm \& sends a message to the operator.

5. Vibration Sensors

If there is any intruder is trying to break-in through the windows, the vibrations of the breakage of glass is measured \& accordingly sends the notification to end user.

6. Contact Sensors

It is used to catch any intruder if the windows are trying to be open or to check the window status. They're divided into two parts; one is connected at the window \& other is at the jamb. If there is no contact between the two parts, it sends a notification to the end user.

7. Android Application

After the completion of the complete system architecture an android application is developed. The Application is paired with Raspberry pi board \& the smart phone by using either Bluetooth, zig-bee or Wi-Fi module using HDMI serial communication.

\section{HARDWARE DESCRIPTION}

For the smart home automation system for monitoring \& controlling of windows, there is the use of various hardware elements, which are listed as below:

\section{Raspberry Pi Board}

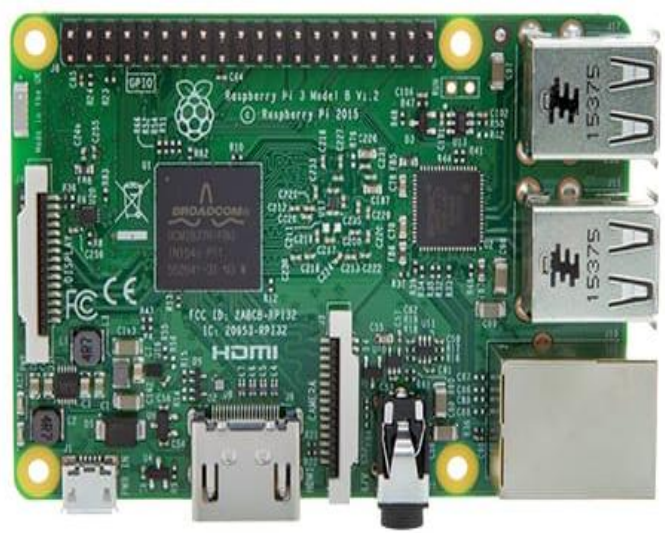

Fig. 4.1 Raspberry Pi Board Type-3

For the system we're using Raspberry Pi board model-3, which is the third generation \& most advanced Raspberry Pi. It is consisting 1.2 GHz quad core processor, Broadcom BCM 2837 SoC ARMv8 CPU with a RAM of 1 GB, having 40 pin GPIO ports. There are also 4 USB ports for the serial interface, 1 full HDMI port for the video output, 3,5mm audio jack, 1 camera interface(CSI), 1 display interface(DSI) \& a micro SD card slot. For the wireless trans-reception antenna is present on board.

The NOOBS operating system is used for the programming for the system by using the programming language Python. For the system raspberry pi is used for the interfacing of both software \& hardware. The IP address of Raspberry pi is interfaced with the IoT over the internet. 
2. DC Motor Driver

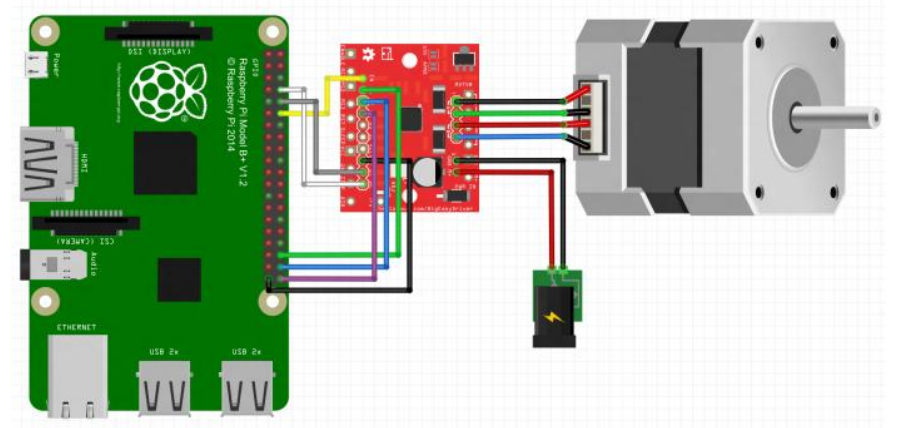

Fig. 4.2 Motor Driver for Raspberry Pi

The L293D is a H-Bridge dc motor driver IC which is interfaced with the controller \& connected at the windows allowing end user to change the windows status. L293D is a 16 pin IC which can control two DC motors simultaneously in both clockwise \& anti-clockwise directions. The L293D is designed to provide bidirectional drive currents of up to $600-\mathrm{mA}$ at voltages from $4.5 \mathrm{~V}$ to $36 \mathrm{~V}$.

\section{PIR Sensor}

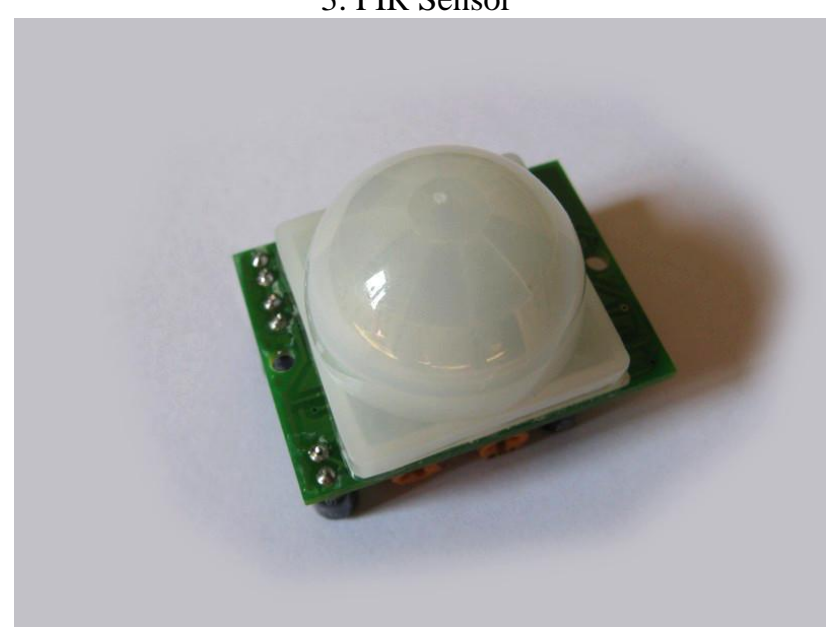

Fig. 4.3 PIR Sensor

PIR sensor is used for the detection of the motion of a person or moving object within the sensors range. According to the movement the sensor sends the information to the user or triggers the burglar alarm inside the house. PIR's basically are passive infrared electric sensors made of pyroelectric sensors, that are used to detect the infrared radiations of the moving objects. It has BISS0001 SoC motion detector IC, operates at $5 \mathrm{~V}-12 \mathrm{~V}$ power rating, to interface with Raspberry pi it consists of a $3.3 \mathrm{~V}$ voltage regulator.

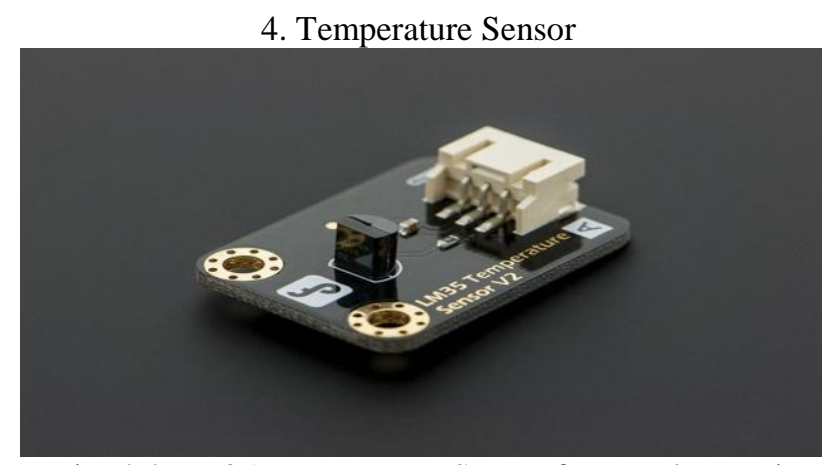

Fig. 4.4 LM35 Temperature Sensor for Raspberry Pi

LM35 Temperature sensors are used to monitor the change in atmospheric condition \& to trigger fire alarm. As the output voltage of LM35 is directly converted in Celsius, it provides fast response. 


\section{OPERATING ENVIRONMENT}

A. Hardware Required

$>$ Raspberry PI 3 Board.

$>$ PIR Sensor.

$>$ Temperature Sensor.

$>$ Contact Sensor.

$>$ DC Motor Driver.

$>$ LED Array.

B. Software Required

$>$ Raspberry OS.

$>$ Python.

$>$ Linux or windows 10 IoT.

$>$ NOOBS.

$>$ JAVA

\section{SYSTEM TESTING}

In this section the system model implementation is to be given,

The various hardware \& software tested outcomes used for system implementation is given,

For the proposed system the main concern is of operating \& controlling of windows, for that a motor driver is used which is interfaced with the controller $\&$ the end user via internet.

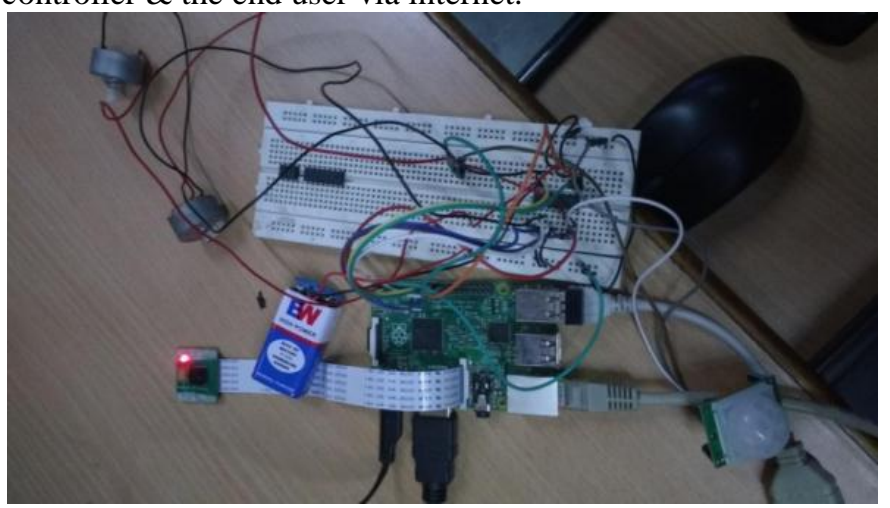

Fig.6.1 Testing of Motor Driver

In above fig. 6.1 we have tested the motor driver, where the signals are sent by the end user from the android application for the changing of windows status and also checks the response time to activate the motor after the signals are sent from application.

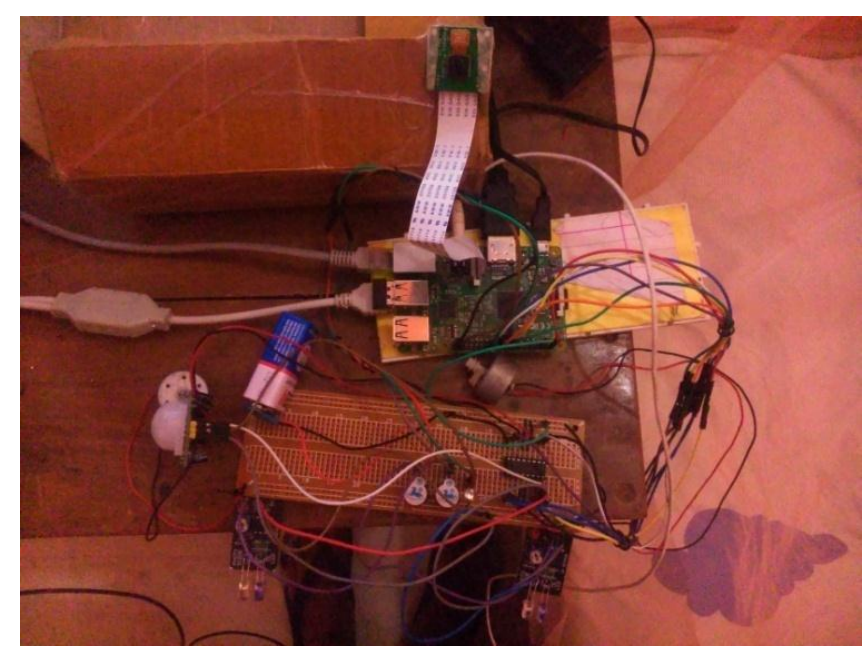

Fig.6.2 Connection on PCB

After the finalization of the functional elements required for the system implementation, all the hardware elements are mounted collectively on a PCB board, as shown in fig. 6.2. 


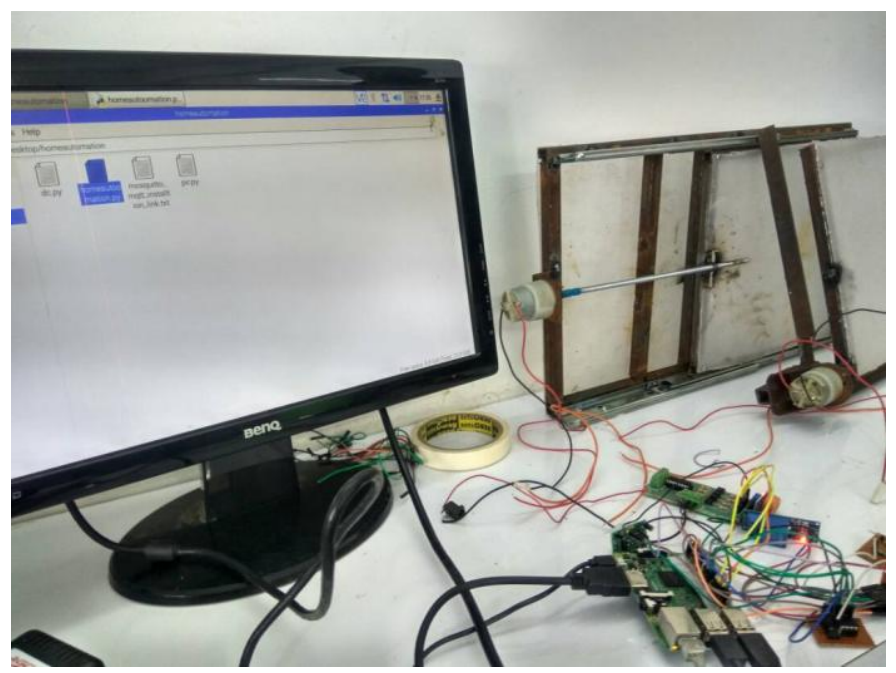

Fig.6.3 Complete System Design

The complete system diagram is shown in the fig. 6.3. where a prototype required for the windows operation \& the total required sensors for system implementation is shown. Along with the raspbarian OS is shown where all of the software part is implemented.

\section{RESULTS}
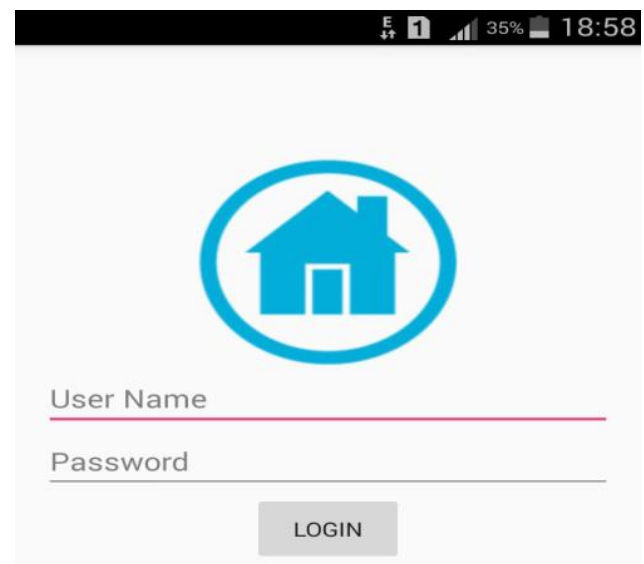

Fig. 7.1 Android Application Home Screen

After the successful connection of the controller to main the server through internet, the data of the sensors are sent to the web server, which is stored online \& can be utilized by the end user. The above figure 7.1 shows the android application which will allows the end user to monitor and control the system through the data present online having unique IP address allocated to the system.

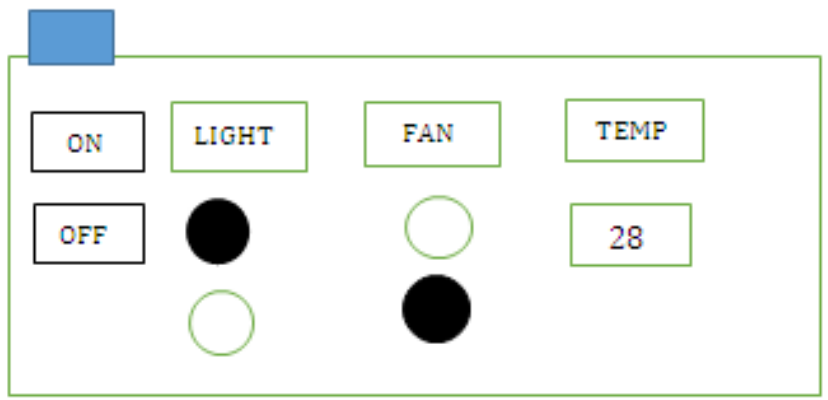

Fig. 7.2 Sensors Data Based Stored in Cloud

All the real time status of all the used sensors are stored online with the use of cloud. This stored data is operated by the end user at any instant of time \& from anywhere through the android application connected via internet with the controller.

Fig.7.2 shows the working condition of lights \& fans inside the smart house, and also shows the present day temperature in Celsius. 


\section{CONCLUSION \& FUTURE SCOPE}

\section{A. CONCLUSION}

In this paper a prototype for the smart home automation \& monitoring for windows using IoT is presented. This system is implemented by integrating relays \& solenoids to raspberry pi board for controlling the windows from a remote location by using an android application. This is the process of controlling \& monitoring of the electrical \& mechanical equipment inside the house. As this automation is based on IoT it provides better integrity of operation \& remotely accessibility. The online interconnection in between the household appliances and the android application makes the home a better, smarter place to live in.

\section{B. FUTURE SCOPE}

As like windows by further programming the doors can also be made smarty accessible over IoT using the android application. Likewise, the smart home automation taking further reference the whole city also can be made smart automated using IoT.

\section{REFERENCES}

[1]. Pavithra.D, Ranjith Balakrishnan" IoT based Monitoring and Control System for Home Automation", 2015 Global Conference on Communication Technologies,IEEE.

[2]. Dongyu Wang, Dixon Lo, Janak Bhimani and Kazunori Sugiura” AnyControl - IoT based Home Appliances Monitoring and Controlling”, IEEE 39th Annual International Computers, Software \& Applications Conference,2015.

[3]. Mr. Pranay P. Gaikwad, Mrs. Jyotsna P. Gabhane, Mrs. Snehal S. Golait” A Survey based on Smart Homes System Using InternetofThings", 2015 INTERNATIONAL CONFERENCE ON COMPUTATION OF POWER, ENERGY, INFORMATION AND COMMUNICATION.

[4]. Byeongkwan Kang,Sunghoi Park,Tacklim Lee and Sehyun Park, "loT based Monitoring System using Tri-level Context Making Model for Smart Home Services", 2015 IEEE International Conference on Consumer Electronics (ICCE), 2015. 